\title{
INVESTIGACIONES
}

\section{Factores e interacciones del proceso de enseñanza-aprendizaje en contextos rurales de la Araucanía, Chile*}

\author{
Factors and interactions of the teaching-learning process in rural contexts \\ of Araucanía, Chile
}

\author{
Rolando Díaz Fuentes, ${ }^{a}$ Sonia Osses Bustingorry, ${ }^{b}$ Sergio Muñoz Navarro ${ }^{c}$ \\ ${ }^{a}$ Facultad de Educación, Ciencias Sociales y Humanidades, Universidad de La Frontera. Temuco, Chile \\ Telf.: (56) 452 592841. Correo electrónico: rolando.diaz@ufrontera.cl \\ ${ }^{\mathrm{b}}$ Departamento de Educación, Universidad de La Frontera. Temuco, Chile \\ Telf.: (56) 452 592841. Correo electrónico: sonia.osses@ufrontera.cl \\ ${ }^{\text {c } D e p a r t a m e n t o ~ d e ~ S a l u d ~ P u ́ b l i c a, ~ U n i v e r s i d a d ~ d e ~ L a ~ F r o n t e r a . ~ T e m u c o, ~ C h i l e ~}$ \\ Telf.: (56) 452 592185. Correo electrónico: sergio.munoz.n@ufrontera.cl
}

\begin{abstract}
RESUMEN
El proceso educativo de niños rurales en la Araucanía, Chile, se desarrolla en contextos socialmente vulnerables, asociando este hecho a que dichos niños obtienen menores rendimientos en pruebas estandarizadas que niños urbanos. Para identificar nuevos aspectos que permitieran mejorar la enseñanza, se utilizó el método de Teoría Fundamentada, hasta la etapa de codificación axial, permitiendo identificar tres factores que fueron denominados como Condicionantes. Estos se relacionaron con la estructura curricular, la enseñanza y las estrategias de aprendizaje de los niños. Este último presentó una importante vinculación con las características afectivas del profesor y con el ambiente natural y sociocultural mediante un aprendizaje significativo y constructivista, aspectos que, de ser utilizados pedagógicamente, pueden mejorar el proceso de enseñanza-aprendizaje y, paralelamente, los rendimientos educativos, permitiendo la mantención del medio ambiente y las tradiciones campesinas e indígenas, bases fundamentales para un nuevo marco curricular de enseñanza para el sistema educativo rural.
\end{abstract}

Palabras clave: docente rural, ambiente rural, aprendizaje significativo, feedback.

\begin{abstract}
The educational process of students from Region de la Araucanía, Chile, is developed in contexts of social vulnerability; this related to the fact that children obtain lower results in standardized tests when compared to urban location students. Fundamental Theory was applied, until its stage of axial codification, in order to identify aspects that could improve the education; this allowed the detection of three factors established as Condicionantes. These factors are related to the curricular structure, the teaching process, and the strategies sued for teaching to children. The latter is strongly linked to the teacher's affective characteristics and to the natural and socio-cultural environment through a meaningful and constructivist learning. These factors, when used pedagogically, improve the teaching-learning process and, at the same time, the school performance. Also generating the maintenance of the environment and the rural and indigenous traditions-building blocks of a new curricular framework for teaching in the rural context educational system.
\end{abstract}

Key words: rural teachers, rural environment, meaningful learning, feedback.

Artículo elaborado en el marco del Proyecto DIUFRO DI15-0082: "Factibilidad socio-educativa y ambiental para el establecimiento de las bases curriculares de un modelo de enseñanza-aprendizaje centrado en el ambiente natural, social y cultural para niños y profesores rurales. Énfasis en la reserva de biósfera Araucarias, región de la Araucanía, Chile". 


\section{INTRODUCCIÓN}

En América Latina y el Caribe existe una significativa relación entre zonas rurales y pobreza, la cual es facilitada por la inequidad de los ingresos económicos que son significativamente más altos en zonas urbanas. En este sentido, las diferentes circunstancias que condicionan la pobreza rural desde un punto de vista socioeconómico hacen difícil concebir soluciones eficaces que permitan mejorar el bienestar de dicha población. Echeverría (2000) señala que debido a factores de desempleo —especialmente en la agricultura- y el insuficiente grado de organización social local, el porcentaje de hogares rurales en situación de pobreza solamente disminuyó de un 56 a un 54 por ciento, mientras que la proporción de población rural en extrema pobreza lo hizo de 34 a 31 por ciento, entre los años 1994 y 1997 (Lakin y Gasperini, 2004).

En tal sentido, la escuela rural no ha sido un factor que permita revertir los bajos índices de pobreza, debido a que históricamente la educación rural no ha tenido una alta prioridad en las políticas nacionales (Hargreaves, Kvalsund y Galton, 2009), siendo afectada por procesos caracterizados por la globalización y los rápidos cambios urbanísticos que han condicionado el currículum y las formas de enseñanza, experimentando reformas que no están dirigidas a solucionar problemas locales, sino que más bien se encuentran en función del capitalismo y el mercado (Hargreaves, 2009; Schafft y Jackson, 2010).

Kvalsund y Hargreaves (2009) sostienen que es importante considerar los conocimientos locales de estos lugares, ya que se encuentran estrechamente relacionados al contexto, ocupándose del cuidado de la naturaleza, la producción de alimentos, la cultura y la vida civil. Dichos autores señalan que, en el caso de países anglosajones, las investigaciones realizadas se llevan a cabo de acuerdo al dominio del sistema externo y a políticas educativas que conducen a desarrollar un determinado tema de investigación, principalmente gestión y administración, lo cual condiciona, a la vez, el modo de investigar, dejando de lado los aspectos particulares de cada escuela.

En Chile, el Ministerio de Educación ha centrado sus políticas educativas de educación rural solamente en la optimización de los recursos fiscales y la gestión escolar, tomando en consideración únicamente resultados de pruebas estandarizadas como el SIMCE (Araya et al., 2012), dejando de lado objetivos primordiales de la educación rural como la superación de la pobreza, la mejora en las condiciones de vida, el desarrollo rural sostenible y la conservación de la cultura local (Atchoarena y Gasperini, 2004).

De acuerdo a las afirmaciones anteriores, es necesario estudiar e identificar elementos particulares que distingan el proceso de enseñanza y aprendizaje de zonas rurales, con el fin de proporcionar nuevos antecedentes que permitan mejorar las políticas educativas y las formas de enseñanza.

\section{MÉTODO}

\subsection{CONTEXTO DE LA INVESTIGACIÓN}

Corresponde a la región de la Araucanía, en Chile. Esta región se divide administrativamente en dos provincias: Malleco y Cautín, y su capital regional es Temuco. La población corresponde a 970419 personas, de quienes un 68\% vive en zonas urbanas y $32 \%$ en zonas rurales. 
La educación rural en la región de la Araucanía se desarrolla en diversos contextos, incluyendo población indígena y no indígena, desde ambientes de montaña y bosque nativo, hasta ambientes desruralizados, representados fundamentalmente por cultivos exóticos; la mayoría presenta dos factores en común: la vulnerabilidad socio-económica de las familias y la despreocupación de los padres respecto de la enseñanza, ambos asociados al rápido proceso de migración campo-ciudad, lo que conduce a las políticas de Estado a dedicar menor atención hacia las escuelas rurales, estableciéndose una educación multigrado, generalmente unidocente, con el fin de optimizar recursos económicos y humanos.

Las personas que participaron de la investigación correspondieron a profesores, directores y jefes de unidades técnico-pedagógicas rurales, con una experiencia que fluctuaba entre 6 y 40 años, y que se desempeñaban tanto en el ámbito municipal como en el particular subvencionado.

\subsection{TÉCNICA DE RECOLECCIÓN DE DATOS}

Correspondió a la entrevista en profundidad, puesto que con ello es posible obtener datos cualitativos profundos expresados por las personas, tales como: formas de pensar y sentir, incluyendo sus valores, motivaciones, deseos, creencias y esquemas de interpretación, aspectos se configuran durante la entrevista (Gaínza, 2006). Se elaboró un guion de entrevista, siguiendo la estructura propuesta por Patton (2002), que incluye preguntas de seis focos: comportamiento y experiencia, opinión y valores, sentimientos y emociones, conocimientos, aspectos sensoriales y demográficos.

\subsection{MÉTODO DE ANÁLISIS}

El método de análisis empleado correspondió a teoría fundamentada hasta la etapa de codificación axial, la cual contempla relacionar las categorías y subcategorías en función de sus propiedades y dimensiones. Según Creswell (1998), esta metodología se fundamenta en el muestreo teórico, la saturación teórica y el análisis de datos mediante el método comparativo constante; según Strauss y Corbin (1998), el primero corresponde a la búsqueda sistemática de nuevos aspectos, identificando causas, condiciones, consecuencias y procesos; el segundo, según Rodríguez, Gil y García (1999), corresponde al criterio que se considerará para la finalización del proceso de selección de los diferentes grupos que tienen relación con alguna categoría de análisis. Por último, el método comparativo constante permite la generación de explicaciones sistemáticas mediante la codificación explícita (segmentación del discurso) y su posterior integración en categorías explicativas para obtener propiedades y dimensiones (Flores, 2009).

\section{RESULTADOS}

De acuerdo a la saturación teórica, se entrevistó un total de 14 profesores de diversas situaciones demográficas, contando, finalmente, con personas de las siguientes comunas: Carahue, Temuco, Padre las Casas, Toltén, Pucón, Lumaco, Los Sauces y Collipulli, de escuelas tanto unidocentes como multidocentes. 
Se obtuvieron 3 categorías: Condicionantes Curriculares, Condicionantes de la Enseñanza y Condicionantes del Aprendizaje; todas, además, con 2 sub-categorías. Se presenta, en primer lugar, los mapas conceptuales que resumen los resultados originados mediante la estructuración y agrupación de los elementos identificados.

\subsection{EL CURRÍCULUM, CONDICIONANTE DEL PROCESO DE ENSEÑANZA-APRENDIZAJE}

Según los profesores rurales, las Condicionantes Curriculares son los factores que más influyen en el sistema educativo y en el aprendizaje de los niños rurales:

...nosotros trabajamos con la ley SEP, entrega un recurso monetario, que va directamente al aula, todos esos recursos a ti te van a permitir innovar en tu práctica pedagógica, tú puedes comprar y llevar al aula, están por ejemplo los computadores, la parte tecnológica, los materiales didácticos y bibliográficos... [Entrevistado 10].

Los detalles de esta condicionante se presentan en la Figura 1 y se describen a continuación.

Figura 1. Categoría, sub-categorías y propiedades de las Condicionantes Curriculares

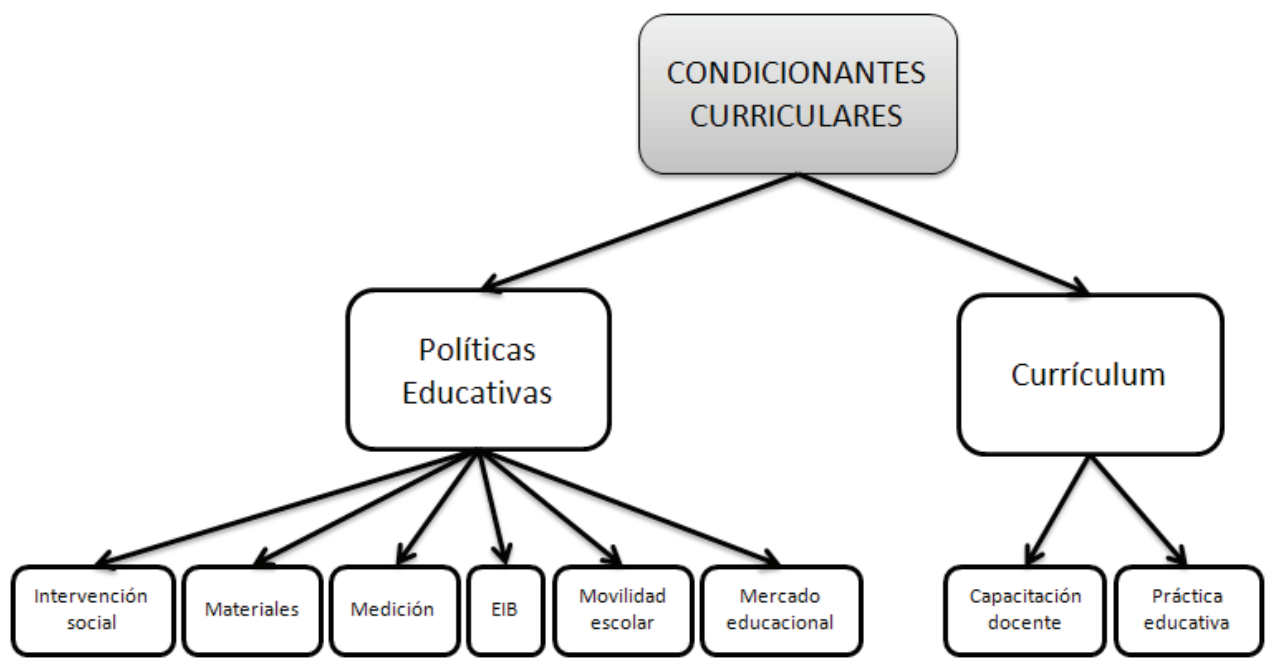

La sub-categoría Políticas educativas considera la variedad de programas involucrados en el sistema educativo, los que tienen por finalidad mejorar las condiciones de vida y atender a las demandas campesinas e indígenas, o, como menciona Duhart (2004), igualar las condiciones de vida rural a la urbana, apuntando a la finalización de la educación y, paralelamente, a la reducción de la pobreza. Esta sub-categoría emerge de la necesidad de mejorar las condiciones sociales de los niños: “...la misión es sacar al niño de donde está, sobre todo en esta área rural, donde el recurso dinero es el que siempre está ausente" [Entrevistado 4]; está directamente relacionado con el ambiente de vulnerabilidad social, 
tanto del niño como del grupo familiar. A nivel mundial, es un objetivo definido en la Cumbre Mundial de la Alimentación (Objetivos Internacionales, 2015), considerando como hecho relevante la desatención de las zonas rurales producto de las tendencias demográficas mundiales y la focalización de la atención en la urbanización (Atchoarena y Gasperini, 2004). Por ello, los contextos rurales han estado sometidos solo a inversión económica, tiempo y experiencia profesional que no han provocado mayores efectos en los índices socioeconómicos, pues su foco es asegurar una correcta gestión educativa, tanto de los actores técnicos como políticos (Erazo, Villalta y Morales, 2010); es así que los esfuerzos realizados en educación rural terminan constituyendo programas de intervención social encubiertos en un proceso educativo, el cual culmina, para el caso de Chile, con la finalización de seis años de escolaridad, lo que mejora los indicadores internacionales y generaría las condiciones necesarias para ofrecer a los niños mejores oportunidades de desarrollo (Echeverría, 2000).

La Ley de Subvención Escolar Preferencial (SEP) es evidencia de lo descrito previamente. Ella exige a las escuelas elaborar un plan de mejoramiento con acciones específicas en las áreas curricular, liderazgo, convivencia y gestión de los recursos, recayendo la responsabilidad en los directores de escuelas (Ahumada et al., 2011). Estos programas se encuentran establecidos en función de resultados medidos a través de pruebas estandarizadas y de la cantidad de estudiantes socioeconómicamente vulnerables, con el fin de mejorar la calidad y su condición de vida. Para ello, algunos materiales proporcionados por este programa juegan un papel importante de apoyo, tanto en la planificación como en el aprendizaje, puesto que mediante guías entregadas por el ministerio se facilita la organización y la planificación del trabajo de aula. Esto es comparable con el modelo Escuela Nueva de Colombia, el cual presenta un fuerte énfasis en la entrega de materiales de autoestudio para las áreas de matemáticas, lenguaje y ciencias (McEwan, 1998); sin embargo, para el caso de la Araucanía, presentaría la falencia docente de no contar con estrategias de autoaprendizaje dirigidas a los estudiantes y de auto-capacitación a los profesores, limitando la aplicación de las guías a los logros descontextualizados de los objetivos curriculares.

Una diferencia importante de la educación rural respecto de la urbana lo constituye la enseñanza intercultural bilingüe (EIB), ya que, como política educativa, es impartida principalmente a primero y segundo año básico en escuelas con alta población indígena (mapuche). Este tipo de programas se ha desarrollado debido, fundamentalmente, a las demandas de los movimientos indígenas, quienes han exigido mejoras educativas en favor de sus culturas (Fernández Droguett, 2005; Stiegler, 2008). Para ello, los estados han tenido que adoptar medidas para una educación de mayor pertinencia cultural, centrándose en la revalorización principalmente del idioma, como una forma de convivencia. Sin embargo la EIB no se encuentra con un total apoyo por parte de las comunidades (apoderados), ya que, en general, estos no desean que sus hijos aprendan de este programa “...se nos ha manifestado en reunión que para enseñar mapudungun a los niños, no lo mandan a la escuela; mejor lo mandan donde el abuelito, hay niños acá en un noventa por ciento que son mapuches..." [Entrevistado 3]. Esto se fundamenta en que este tipo de enseñanza obstaculizaría a los niños mapuche aprender las materias de la cultura occidental, cultura en la cual se desenvolverán y desarrollarán posteriormente como personas. Lo anterior demuestra que la EIB no ha sido debidamente implementada y desarrollada en áreas rurales, ya que se contrapone a casos exitosos mencionados por estudios internacionales donde se involucra en el trabajo educativo a la comunidad en general alcanzando a la escuela, las familias y los estudiantes, remitiéndose no solo a la enseñanza en aula, sino que en directo 
contacto con el medio ambiente y su cultura; según Abadzi (2008), solo de esta manera es posible mejorar los aprendizajes, lo cual puede lograrse fundamentalmente a edades tempranas. Esto se suma a lo declarado por UNESCO (2009) que señala que una educación culturalmente pertinente debe considerar la elaboración de planes y programas de estudio encaminados a aumentar la pertinencia de la educación mediante el ajuste de los procesos de aprendizaje, los contenidos educativos, la capacitación de los docentes y la gestión escolar.

Por otro lado, las Políticas educativas rigen la práctica educativa rural, condicionándola mediante la aplicación y los resultados de la prueba estandarizada SIMCE. Esta es aplicada censalmente en Chile, a objeto de medir y evaluar los avances educativos nacionales, por lo que según sus resultados se proponen y reestructuran las políticas educativas, permitiendo perfeccionar la enseñanza (Cerón y Lara, 2011). Este tipo de evaluación promueve la generación de indicadores del aprendizaje que favorecen la comparación entre establecimientos educativos, convirtiendo la educación en un sistema de libre mercado donde los apoderados, transformados en clientes, pueden conocer y comparar resultados para elegir la escuela adecuada para sus hijos, mediante la reputación local de cada escuela. En otros países, por ejemplo, estos mecanismos son validados a través de los denominados "League Tables", indicadores educativos que permiten hacer comparaciones objetivas respecto de la calidad de la enseñanza, mecanismo con el que no se cuenta en el sistema de educación chileno (Aguerrondo, 2008).

Otro aspecto que contribuye a la mercantilización de la educación en escuelas rurales corresponde a las distintas estrategias comerciales utilizadas por algunos establecimientos rurales "...te voy a dar un quintal de harina, y te voy a dar una caja de mercadería [...] lo va a buscar un vehículo, y se enojó el apoderado con ese sostenedor porque no le ofreció otra cosa, entonces lo cambia..." [Entrevistado 2]. De aspectos como estos se desprende la propiedad movilidad escolar, la cual, según los docentes rurales, considera la movilidad de los niños entre diferentes escuelas producto de los diferentes beneficios ofrecidos por estas, especialmente por las escuelas particulares subvencionadas que contribuyen con alimentación, transporte, internado o ropa, aspectos que permiten a las familias atenuar sus carencias económicas facilitando a las escuelas reunir mayor matrícula y sustentar de esta forma el negocio educativo. Según lo investigado, las escuelas municipales también han comenzado a replicar dicha dinámica; sin embargo, les dificulta un poco más este aspecto, pues la gran mayoría de ellas corresponde al tipo multigrado y unidocente. Del mismo modo, los apoderados estiman que la enseñanza en estas escuelas es de menor calidad, y prefieren las escuelas con cursos simples. Esto ocasiona que muchos establecimientos vayan quedando sin matrícula, lo que desencadena el cierre de la escuela, especialmente de las más alejadas de las ciudades; frente a ello los escasos niños y sus familias deben emigrar a otros lugares, generalmente urbanos, perdiendo los territorios, la reproducción cultural y la lengua indígena, lo que según Williamson (2004) atenta contra los derechos humanos y los derechos de los niños por no reconocer la prioridad del aprendizaje en su lengua materna y en su medio cultural de origen.

En la sub-categoría Currículum, los profesores mencionan: “...partimos con los objetivos generales y empezamos a resumir y ver los aprendizajes esperados, los calendarizamos por semana, por días y los planteamos por mes, de esa manera vamos revisando..." [Entrevistado 14], se describen los aspectos formales que regulan la labor docente, y sus propiedades son capacitación docente y práctica educativa. La primera determina, en mayor o menor grado, la especialización docente para impartir las materias y adecuarse a los cambiantes marcos curriculares, permitiendo a los docentes 
mejorar sus planificaciones y sus prácticas educativas, ya que les proporciona una mejor base conceptual y el logro de los objetivos educativos; esto último posibilita, a su vez, la generación de redes de trabajo y colaboración, principalmente las establecidas en el espacio del microcentro, facilitando la profundización de conocimientos y la comprensión de directrices gubernamentales, lo que permite enfrentar de mejor manera las deficiencias docentes provenientes de la formación inicial, asimismo permite enfrentar y tomar medidas respecto del ambiente de vulnerabilidad, la precariedad de las escuelas y los bajos niveles de aprendizaje, de acuerdo a las distintas mediciones realizadas (Moreno, 2007).

Desde la experiencia y creencias de los docentes rurales, la práctica educativa se centra en una perspectiva contextual, en la cual es considerado principalmente el desarrollo del lenguaje, la lectura y la disciplina, aspectos que constituirían, a modo de ver de los profesores, una educación de calidad. Esta contextualización, según Vera Bachmann, Osses y Schiefelbein (2012), tiene un importante efecto en el proceso educativo rural, pues al realizar este tipo de enseñanza, el docente pasa a desempeñar el rol de mediador entre el niño y su entorno, transformándolo en una importante variable dentro del proceso educativo, donde son considerables las carencias cognitivas y afectivas de los niños.

\subsection{FACTORES QUE CONDICIONAN LA ENSEÑANZA}

Según los profesores rurales, las Condicionantes de la Enseñanza son las que inciden directamente en la práctica pedagógica, y se encuentran también asociadas con aspectos afectivos "...lo que manda en la ruralidad es la parte maternal, la parte afectiva, si yo soy activa con el niño, si no tengo pegamento, hago engrudo, algo haré para que el chico tenga sus materiales y pueda trabajar, yo creo que para mí el resultado es la afectividad y el cariño que los niños sienten..." [Entrevistado 1]. Los detalles de esta condicionante se presentan en la Figura 2 y se describen a continuación.

Figura 2. Categoría, sub-categorías y propiedades de las Condicionantes de la Enseñanza. t corresponde al tiempo, propiedad dinámica de la práctica educativa

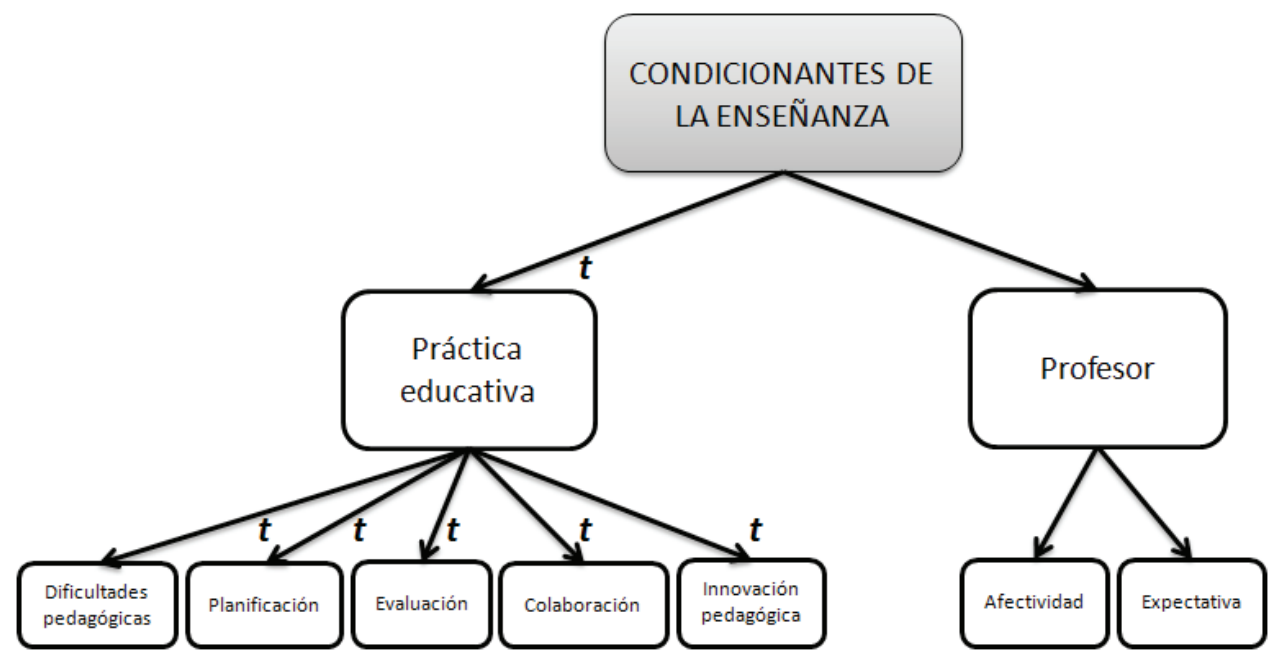


Desde las Condicionantes de la Enseñanza es posible distinguir los aspectos que involucran al docente rural como persona. Juega un rol importante la experiencia profesional que revaloriza la profesión docente mediante las características personales, la formación vocacional y las creencias. Dichos aspectos se obtienen de citas como: “...no lo que voy a ganar, no en que yo tengo que entrar a las 9 y salir a las 4, yo creo que un compromiso como educador es mucho más allá, [...] si el alumno necesita que le explique algo hasta más tarde, yo me quedo y lo hago" [Entrevistado 8]; se desprende, entonces, la sub-categoría Profesor, la cual representa los aspectos interrelacionados afectividad y expectativa que el profesor trasmite consciente e inconscientemente a los estudiantes, generando con ellos lazos afectivos que promueven y refuerzan el aprendizaje, haciendo efectivas las prácticas educativas. Aquí son fundamentales las emociones que, al ser consideradas, potencian el proceso de aprendizaje (Villarroel y Leiva, 2005). Estos aspectos, a pesar de no contar con la formación y las competencias necesarias para un trabajo psicosocial y/o comunitario, son desarrollados por el docente rural, quien conoce las carencias del entorno familiar y social de los niños, haciéndolos parte de su práctica educativa.

Estrechamente relacionada con la familia, la propiedad expectativa describe la visión de vida futura que posee el docente rural hacia los estudiantes, contraponiéndose con la expectativa del estudiante y del grupo familiar que se origina, principalmente, a partir de la escasez de recursos económicos, factor de mayor influencia en el aprendizaje (Cornejo y Redondo, 2007), esto porque limita las posibilidades de conseguir, por parte del grupo familiar, una educación de calidad. Por lo tanto, la expectativa entregada por el profesor a sus estudiantes es importante porque estaría dada por su propia experiencia; es decir, la retrospección de su propia vida, las dificultades que ha superado otorgan al profesor un insumo importante para transmitir a los niños. Esto se debe a que profesores rurales provienen generalmente de estratos sociales bajos, principalmente de clase media, obrera y campesina (Mizala et al., 2000).

La segunda sub-categoría, Práctica educativa, surge de citas como: “...bien comprometida con todos los colegas, tratan ellos de intercambiar trabajos, se está trabajando mucho con guías, con sus planificaciones al día y lo vamos conversando entre nosotros..." [Entrevistado 9], esto hace mención a la labor y al compromiso del docente en el aula. Para estas zonas desfavorecidas socialmente, se pudo advertir que se otorga mayor relevancia a la etnicidad a través de la enseñanza de la lengua (mapudungun), debido a la importante concentración de personas de la etnia mapuche (provincia de Malleco) y a las demandas que han realizado comunidades y organizaciones territoriales al Estado de Chile; en estas zonas se observa claramente un fuerte sentido de pertenencia cultural, el cual se manifiesta en el discurso docente y la relación etnia-pobreza.

La propiedad dificultades pedagógicas de la sub-categoría Práctica educativa alude a los obstáculos que influyen durante el desarrollo de la práctica educativa. Se refieren a dos ámbitos: las dificultades de aula (relacionadas con la formalidad curricular) y las dificultades familiares. Las primeras se relacionan con los extensos horarios de trabajo que obstaculizan la planificación de las actividades, las que se realizan generalmente para distintos niveles y edades (cursos multigrados), con falta de materiales adecuados (descontextualizados); las segundas, en tanto, corresponden principalmente a la escasa comunicación de los niños con sus familias, problemas psicológicos, de salud (ausentismo escolar) y de lejanía a las escuelas (Echeverría, 2000). Algunas veces estas dificultades son atenuadas con apoyo de otros profesores (trabajo en red en los microcentros) o la 
incorporación de otros profesionales, cuando se dispone de fondos para ello (Ley SEP). Esto último ha permitido, mediante la adquisición de nuevos materiales y la contratación de profesionales principalmente del área de la psicología, mejorar aspectos relacionados con la planificación, mediante la cual se facilita la incorporación de otras actividades como la enseñanza intercultural, la realización de actividades extracurriculares y el involucramiento de la familia, optimizando así el tiempo de planificación. El solo hecho de no contar con dichos apoyos, junto a la implementación de la enseñanza intercultural bilingüe, la evaluación, la supervisión docente, más la preparación SIMCE, se convierten en obstáculos para la enseñanza y el aprendizaje.

Es importante señalar que las dificultades pedagógicas van entregando al docente rural la experiencia necesaria para contrarrestarlas, mejorando de esta forma su práctica para la obtención de mejoras educativas; este aspecto se logra mediante la innovación pedagógica, la cual consiste en incorporar en la planificación y en la práctica educativa diferentes aspectos que el docente cree son importantes para el aprendizaje, destacándose principalmente la contextualización, la cual genera aprendizajes significativos y la conservación cultural mediante una retroalimentación de aspectos propios de la comunidad, tanto del ambiente natural como del ámbito socio-cultural y económico.

Para ello se considera la opinión y la forma de ver el mundo de los propios estudiantes y la opinión de los padres, quienes ya cuentan con la experiencia de su contexto: “...se hace con padres, apoderados y alumnos, igual cuando se hacen clases de ciencia, se sale afuera, al entorno, hacen encuestas, por decir al jefe de una comunidad..." [Entrevistado 1]. Algunas veces estas actividades se acompañan de actividades artísticas o tareas de desarrollo junto a los padres, actividades de problematización de diferentes situaciones del territorio o salidas a terreno que pretenden dar respuesta a alguna inquietud de la comunidad, enfocándose hacia la acción. Acá juega un papel fundamental la propiedad "Colaboración", la cual emerge de citas como: “...el compromiso de los padres también es mayor, porque nosotros tenemos el consejo escolar y que se forma al comienzo del año y en ese consejo, uno les está informando de todo..." [Entrevistado 5]. Se desprende de esta cita que la práctica educativa intenta generarse colaborativamente entre el docente y las familias; de esta forma, mediante su experiencia y transparencia logra la confianza de los apoderados que terminan participando en las labores de aprendizaje, lo que transforma al profesor rural en un referente local.

Para la Práctica educativa se cuenta también con la evaluación, consistente en la medición de los aprendizaje, la cual se efectúa principalmente de manera sumativa (pruebas escritas), debido a que constituyen requisitos curriculares. Sin embargo, dichas evaluaciones no permiten abarcar la heterogeneidad del aprendizaje escolar, por lo que docentes rurales, especialmente quienes poseen más experiencia, realizan evaluaciones diferenciadas según las capacidades de los niños (diagnóstica, evaluaciones de proceso, evaluaciones subjetivas y, ocasionalmente, autoevaluaciones de los propios estudiantes). No obstante, estos tipos de evaluación no forman parte de la planificación, sino que se efectúan solo como parte de una evaluación personal que permite reflexionar sobre la práctica educativa y ponderar las notas sumativas a objeto de no perjudicar el avance curricular de los niños. Un aspecto importante de la evaluación son las acciones que realizan algunos docentes con sus estudiantes una vez egresados; se trata de un seguimiento informal a las calificaciones de sus ex-estudiantes en la nueva escuela, permitiendo hacer una valoración de su práctica pedagógica, de modo tal que facilite la adopción de medidas correctivas o la reorganización de las lecciones respecto de su práctica educativa. 
Finalmente, una limitante importante de la enseñanza es el tiempo, factor que Huitt (2006) considera esencial, porque es llevado a cabo dentro de la sala de clases, asociándolo con la planificación de las lecciones. Estas actividades demandan una gran cantidad de horas para su desarrollo; es así como el tiempo necesario para el trabajo de aula no considera, por ejemplo, las heterogéneas capacidades de los estudiantes, por lo que resulta contradictoria la extensión y la descontextualización curricular que provoca un desequilibrio cognitivo en los niños. Por ello, el desarrollo de lecciones de carácter constructivista (participativo y colaborativo) que algunas veces realiza el docente rural permite optimizar el tiempo, principalmente el de planificación, ya que un aprendizaje de este tipo va construyendo de forma paulatina el objeto de estudio, lo que sumado al bagaje cultural de los niños y el de sus familias, favorece la motivación y reduce la frustración, factores que aceleran el fracaso escolar (Ruíz de Miguel, 2001).

\subsection{FACTORES QUE CONDICIONAN EL APRENDIZAJE}

La categoría Condicionantes del Aprendizaje (Figura 3) describe algunas características personales de los niños y aspectos ambientales específicos que influyen en él: “... el alumno que tengo es de escasos recursos, siempre tiene hambre, siempre tiene apetito, siempre tiene carencias afectivas..." [Entrevistado 7].

Figura 3. Categoría, sub-categorías y propiedades de las Condicionantes del Aprendizaje. t corresponde al tiempo y es propiedad dinámica del ambiente

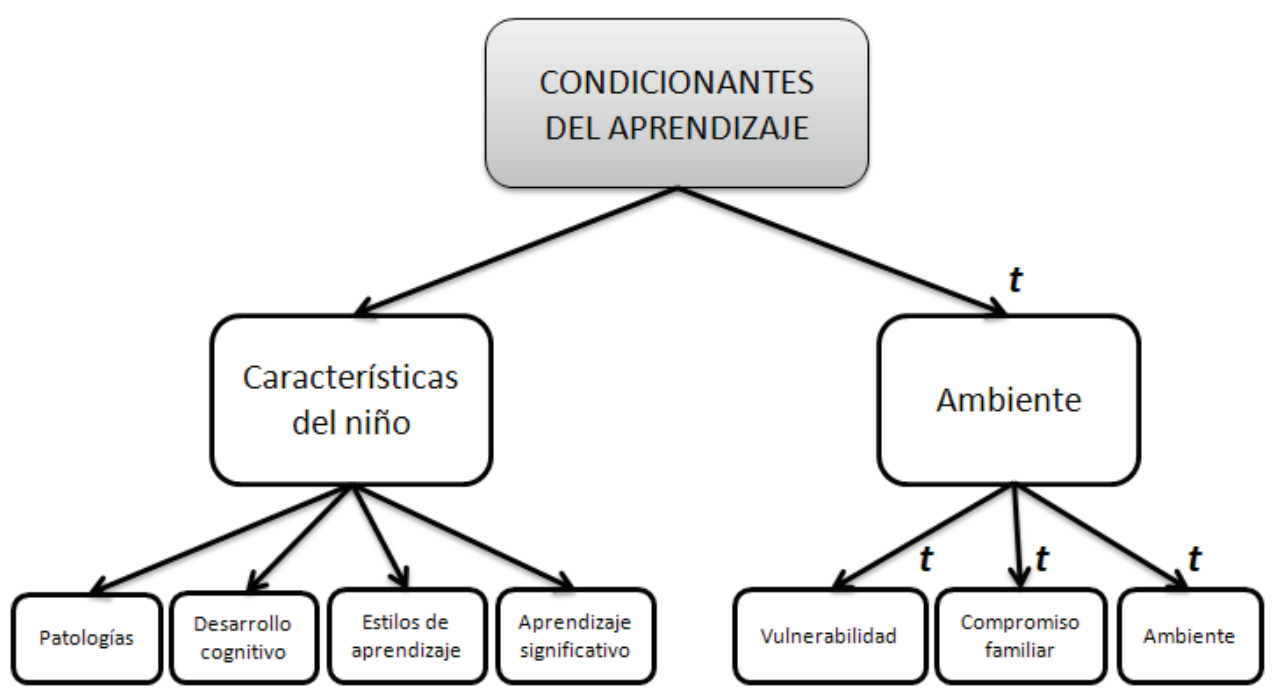

Los elementos de esta categoría son considerados por los docentes rurales en las prácticas educativas como estrategias que aminoran las limitaciones de vulnerabilidad: “... estamos pasando una unidad de geometría y la trabajamos casi más fuera que dentro de la sala, las dimensiones de los árboles; en cuanto a lo que ellos comercializan por ejemplo, 
[...] el pescado, y de ahí llevarlo a otras cosas, así lo relacionan bastante" [Entrevistado 6]. La sub-categoría, Características del niño considera los aspectos más influyentes en su aprendizaje, entre ellos patologías que limitan la asistencia a clases (Schiefelbein y Simmons, 1981), las más comunes de mencionar son: malnutrición, dislexia, dislalia y algunos trastornos psicológicos que debieran haber sido atendidos en educación pre-escolar. Este tipo de educación con que los niños rurales no cuentan acrecienta los problemas a medida que se avanza en edad, repercutiendo fuertemente en el desarrollo cognitivo. A esto se suma la falta de preparación profesional de los docentes para atender este tipo de necesidades, lo que limita el aprendizaje, requiriendo, por tanto, la articulación de profesionales de centros de salud local o el apoyo específico de profesionales de dichas áreas. Del igual modo, desde el aula el aprendizaje se encuentra influenciado por los rigurosos y estructurados contenidos curriculares, considerados por los propios niños como sin sentido. Esta desconexión entre la realidad del niño y el aula provoca una desorganización cognitiva que limita aún más el desarrollo cognitivo, especialmente algunas habilidades básicas del pensamiento como: describir, comparar, relacionar y comprender. Para sobrellevar estas deficiencias, autores como Rudduck y Flutter (2004) aconsejan la utilización de aspectos personales y ambientales de los niños, tales como sus visiones y anhelos, los cuales son importantes a la hora de considerar una enseñanza contextualizada.

Dentro del contexto de aprendizaje, se conoce que los niños pueden aprender a través de la "mediación", principalmente la que se desarrolla a través de la tecnología, momentos en los cuales el niño, de manera inconsciente, desarrolla diversas estrategias que le facilitan su aprendizaje. Este tipo de aspecto fue denominado en el presente estudio como estilos de aprendizaje, el cual corresponde al proceso de adoptar estrategias particulares de procesamiento de información, sin importar las exigencias específicas de una tarea (Bermeosolo, 2007). Se fundamenta en los sentidos, la práctica concreta, la interacción con el medio, los valores y, en menor grado, con los contenidos curriculares cuando estos son desarrollados estratégicamente mediante el aprender haciendo y el descubrimiento guiado.

Para el desarrollo del aprendizaje de niños rurales son importantes las actividades cotidianas desarrolladas por las familias, en las cuales el niño activamente participa: “...por alguna necesidad de la casa, los chicos tienen que hacer cosas, porque la mamá no tiene los medios económicos; mediante el trabajo, los chicos van aprendiendo por necesidad." [Entrevistado 4]. Por lo tanto, son importantes las actividades económicas y culturales, dentro de las cuales se pueden señalar la comercialización de productos provenientes del campo, actividades culturales indígenas o actividades hogareñas como la colecta de huevos, obtención y trasporte de agua de un pozo, el conteo o rodeo de animales, y todas las dimensiones micros y macros de cada fase de la vida que el niño desarrolla y socializa (Edwards et al., 2006). De esta manera, el aprendizaje se inicia y refuerza mediante aspectos que al niño le gustan y le importan, siendo estos necesarios y significativos para el bienestar del hogar.

Según Bermeosolo (2007), dichas actividades se relacionan con el estilo identificado por Marton y equipo como de tipo deep-level, que enfatiza la sensibilidad frente a las demandas situacionales y la importancia de las exigencias ambientales mediante las cuales los resultados del proceso de aprendizaje se orientan a las conclusiones, lo que implica una evaluación del argumento y sus fundamentos, permitiendo al niño relacionar las ideas con sus experiencias personales. Según esto, es importante para el aprendizaje de los niños rurales el concepto de aprendizaje significativo, el cual se describe como el aprendizaje que 
el propio individuo descubre e incorpora por sí mismo, tanto en la vida como en la escuela, así muchos aspectos resultan no enseñados, sino aprendidos, como menciona Zárate (2002): "Aprender es atesorar en la mente por medio del propio pensamiento una nueva idea o verdad" (p. 21). Por ello, el docente rural observa la realidad ambiental del niño y los distintos fenómenos significativos que contribuyen a su aprendizaje, relacionando las personas de la comunidad y los distintos elementos del medio, a fin de hacer interesante y propio el objeto de conocimiento.

Según lo señalado anteriormente, aparece la sub-categoría ambiente, que corresponde al medio circundante donde el niño rural vive y se desarrolla. Tiene como propiedad su vulnerabilidad, expresada en las carencias sociales y económicas que poseen las familias, dentro de las cuales son importantes las carencias afectivas y nutricionales, asociadas con el contexto de pobreza. Para mejorar esta condición, los niños rurales participan activamente en las distintas actividades económicas de las familias, jugando un rol fundamental el compromiso familiar que se diferencia de la propiedad colaboración descrita en la categoría de la enseñanza, en que no tiene relación con la práctica educativa desarrollada por el docente, sino que más bien se relaciona con el grado en que la familia anima el aprendizaje para salir de la pobreza. Este aspecto en particular busca romper de cierta forma el círculo de dependencia existente entre la disponibilidad económica de los padres y el nivel educativo de ellos (Schiefelbein y Simmons, 1981).

Si bien en lo anteriormente descrito destacan las dificultades y deficiencias del sistema de educación rural, dentro de la experiencia docente el ambiente pasa a ser el factor más importante en el proceso de enseñanza-aprendizaje, ya que ofrece variados elementos asociados a actividades pedagógicas que facilitan el aprendizaje, tales como: salidas a terreno, encuentros intergeneracionales o el trayecto de la casa a la escuela. Estos aspectos son descritos por Stables (2007) como circundantes de la escuela, porque se encuentran asociados a los ámbitos socio-culturales, económicos y naturales, espacios en los cuales claramente la familia se manifiesta en forma comunitaria. Entre estos cabe mencionar los relacionados con la etnia mapuche, los que presentan importantes aspectos de colaboración y solidaridad, especialmente en lo que se refiere al intercambio de diferentes bienes como los producidos o recolectados en el campo; mientras que desde el punto de vista natural, es importante la relación niño-naturaleza que se materializa a través de la observación, principalmente relacionada con animales los cuales manifiestan diversos comportamientos que exigen explicación por parte de ellos. Estos aspectos organizados inconscientemente en la mente son usados en distintas actividades de enseñanza y en la planificación de estrategias, puesto que facilitan el aprendizaje haciéndolo significativo (Páramo, 2007).

Estos recursos ambientales, facilitadores del aprendizaje, corresponden a distintas interacciones entre el individuo y su medio, los cuales permiten a las personas poseer un dominio práctico. Por ello, es común por parte del docente rural, el desarrollo de actividades al aire libre, ya que permiten reforzar aspectos del lenguaje y las matemáticas, relacionándolas especialmente a actividades asociadas con las ciencias, dado que dicha asignatura tiene mayor relación con el ambiente, especialmente los aspectos del desarrollo de la vida, la reproducción de animales y plantas, la tierra, el universo, el cuerpo humano y la salud, a través de los cuales los niños exploran, clasifican, analizan, comparan, comunican, evalúan, experimentan, formulan preguntas y miden objetos, todas actividades contenidas en las bases curriculares, que pueden ser trabajadas en su ambiente para el logro de aprendizajes significativos. 
Para lo anterior juega un rol importante la Educación Intercultural Bilingüe, ya que mediante un asesor intercultural se facilita la contextualización de los contenidos, dando significancia a la lengua y revalorizando los elementos del ambiente, tanto culturales como ambientales y sociales, siendo para ello necesario considerar el factor tiempo como limitante de la práctica educativa. Según Carroll (1963) el tiempo es distribuido por el niño en las distintas actividades de su vida, y es posible descomponerlo en tiempo gastado en aprender y tiempo necesario para aprender. En este caso, el primero corresponde a la motivación asociada a la disponibilidad de los recursos (elementos del ambiente) y el segundo a la aptitud, es decir, la capacidad de aprender y la calidad de la enseñanza (lograda mediante distintas estrategias para lograr los aprendizajes esperados). Esto transforma el proceso de enseñanza-aprendizaje en un complejo y dinámico sistema interrelacionado que varía a cortas fracciones de tiempo, pudiendo ser utilizado de manera óptima para el aprendizaje (Carroll, 1993).

\section{DISCUSIÓN}

Según la Real Academia Española, la palabra ambiente es definida como las condiciones o circunstancias físicas, sociales y económicas de un lugar, de una reunión, de una colectividad o de una época. Para el niño rural, el ambiente se compone de su familia, su grupo socio-cultural, su escuela y el mundo físico, este último, está constituido por componentes bióticos y abióticos. Entre estos componentes y las personas existe una interacción dinámica que, junto a su propio conocimiento, permite al niño almacenar, organizar y reconstruir imágenes y aprendizajes que se manifiestan en el comportamiento (Holahan, 2008). La Figura 4 representa la relación e integración dinámica de los distintos componentes del sistema, indicando la fuerza de asociación entre dichos componentes.

Las variables del ambiente inmediato forman parte importante del aprendizaje, porque están directamente ligadas entre sí mediante una retroalimentación (feedback) que se manifiesta a través de la actitud, la conducta y el aprendizaje. Gibson (1986) señala, en primera instancia, que el ambiente posibilita el descubrimiento de significados que permiten mantener una cierta inquietud en los niños, provocando su interés en aprender; y, en segunda instancia, Echarri y Puig I Baguer (2008) señalan que favorece la comprensión y consiguiente protección, respeto y valorización del ambiente que los rodea. 
Figura 4. Elementos e interacciones del sistema de educación rural, región de la Araucanía.

Línea continua: relación fuerte; línea discontinua: relación media; línea punteada: relación débil y donde t corresponde al tiempo y es propiedad dinámica en las condicionantes de la enseñanza y el aprendizaje

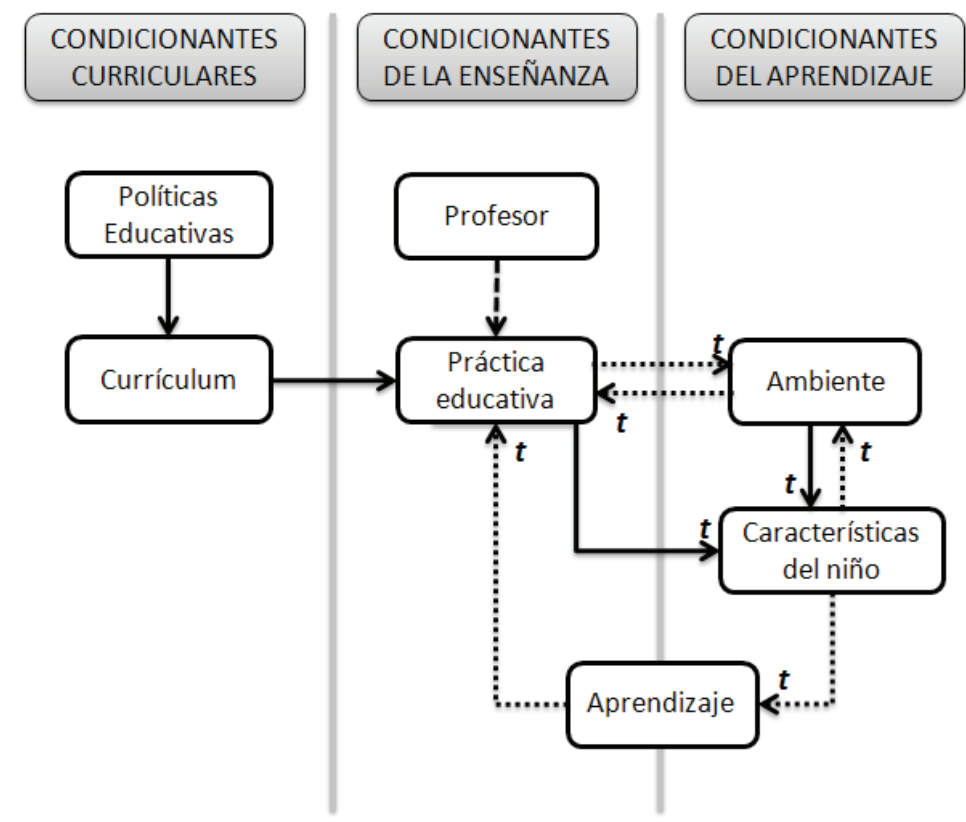

Páramo (2010) sostiene que el ambiente físico, asumido como espacio público transformado en lugar de aprendizaje, ejerce una influencia importante al establecer reglas sociales y, como tal, constituye el lugar donde se exhiben las prácticas sociales que identifican y cohesionan los grupos sociales. Por otro lado, Maturana y Varela (2002) sostienen que el entorno físico permite la construcción de conocimientos a través de cualquier perturbación del medio que afecte en sí mismo al ser vivo, por tanto, el individuo es quien determina su propio cambio. Este proceso, según dichos autores, no sería instructivo, ya que no se pueden determinar sus efectos. Por ello, la interacción educativa sujeto-entorno permite que los aprendizajes sean constructivos mediante un feedback que revalora la estructura semántica del individuo que aprende, reestructurando la comprensión y preparando la mente y el sistema cognitivo para un nuevo aprendizaje (Novo, 1995). Esto sucede porque el aprendizaje no se desarrolla secuencialmente, sino que se genera mediante diferentes etapas de acreción, las cuales van constituyendo nuevas estructuras cognitivas que permiten la sintonía (aprendizaje), es decir, una reestructuración cognitiva complejizada (Engeström y Sannino, 2012). En la práctica, esto es aprovechado inconscientemente por el docente rural, quien hace de mediador entre el ambiente y el niño, colocando en práctica su experiencia y su conocimiento para utilizar distintos componentes del ambiente.

Por otro lado, el ambiente escolar — espacio constituido por la familia, la cultura, la escuela y el ambiente natural - ofrece significados individuales y compartidos que conforman el escenario de vida, permitiendo la construcción, transformación y el 
fortalecimiento del espacio educativo, revalorándolo y desarrollando su propia identidad (place identity). En su interior hay variados factores que influyen en las actitudes, la participación y el aprendizaje, lo que constituye, a nivel internacional, una nueva corriente de investigación denominada aulas abiertas (open classroom) (Gilmartín, 2009). En esta corriente es posible encontrar orientaciones para el estudio del ambiente escolar, definido como todos los espacios emplazados en un contexto socio-ambiental que al integrarse facilitan la mediación, el aprendizaje y la incorporación de estímulos que provocan respuestas de carácter conductista (Travers, 1976). Estos mediadores, no considerados conscientemente por las personas, facilitan, por ejemplo, la resolución de operaciones matemáticas que pueden ser mediadas por cualquier actividad doméstica realizada por el niño rural; si bien este proceso es condicionado, el rol del docente es transformar este mecanismo pasivo en uno activo y constructivista que permita la mantención de los aspectos socioculturales, económicos y ambientales, los que deben ser trabajados desde una lógica de desarrollo sustentable, manteniendo las características ambientales y su identidad.

\section{COMENTARIOS FINALES}

La concepción de los profesores rurales muestra una alta valoración del currículum nacional, ya que establece las normas ministeriales que rigen la práctica educativa; sin embargo, dichas normas limitan el desarrollo de actividades pedagógicas contextualizadas, las que aparentemente son la fuente de aprendizaje de los niños rurales. Este hecho se fundamenta en aspectos de psicología ambiental que deben ser considerados en la planificación de las actividades educativas, como asimismo al momento de realizar las evaluaciones estandarizadas, las cuales pueden ser fuente de importantes sesgos.

El proceso de enseñanza-aprendizaje realizado con niños de escuelas rurales debe desarrollarse desde las características particulares del ambiente, porque el proceso de interacción dinámica aprendizaje-ambiente (Feedback) permite que los aprendizajes sean significativos, los que a su vez contribuyen a la valoración del territorio y de los elementos que interactúan en él, facilitando la construcción de aspectos sociales, ambientales y económicos que componen la identidad, fortaleciendo la pertenencia cultural.

\section{REFERENCIAS BIBLIOGRÁFICAS}

Abadzi, H. (2008). Orientaciones para el desarrollo. Aprendizaje eficaz y pobreza: Ideas desde las fronteras de la neurociencia cognitiva. Santiago, Chile: Ediciones UCSH.

Aguerrondo, I. (2008). La influencia del contexto en la efectividad de la escuela. Consideraciones para el desarrollo profesional docente. En R. Blanco (Ed.), Eficacia escolar y factores asociados en América latina y el Caribe (pp. 61-96). Santiago, Chile: Oficina Regional de Educación de la UNESCO para América Latina y el Caribe (OREALC/UNESCO Santiago) y el Laboratorio Latinoamericano de Evaluación de la Calidad de la Educación (LLECE).

Ahumada, L., Sisto, V., López, V., \& Valenzuela, J. (2011). Liderazgo distribuido durante el proceso de elaboración e implementación de planes de mejoramiento educativo en una comuna rural en Chile. Revista de Ciencias Sociales, 17(2), 262-273.

Araya, F., Dell, R., Donoso, P., Marianov, V., Martínez, F., \& Weintraub, A. (2012). Optimizing location and size of rural schools in Chile. International Transactions in Operational Research, 
Estudios Pedagógicos XLII, N 3: 111-128, 2016

FACTORES E INTERACCIONES DEL PROCESO DE ENSEÑANZA-APRENDIZAJE EN CONTEXTOS RURALES DE LA

ARAUCANÍA, CHILE

19, 695-710.

Atchoarena, D., \& Gasperini, L. (2004). Educación y desarrollo rural: definición del marco de referencia. En D. Atchoarena y L. Gasperini (Eds.), Educación para el desarrollo rural: hacia nuevas respuesta de política (pp. 27-80). Roma, Italia: FAO/UNESCO-IIEPE.

Bermeosolo, J. (2007). Como aprenden los seres humanos. Mecanismos psicológicos del aprendizaje (2da ed.). Santiago, Chile: Ediciones Universidad Católica de Chile.

Carroll, J. (1963). A model of school learning. Teachers College Record, 64, 723-733.

Carroll, J. (1993). Human cognitive abilities. A survey of factor-analytic studies. Nueva York, USA: University of Cambridge.

Cerón, F., \& Lara, M. (2011). Factores asociados con el rendimiento escolar SIMCE 2010, Educación Matemática $4^{\circ}$ Básico e Inglés $3^{\circ}$ Medio: Ministerio de Educación, Gobierno de Chile.

Cornejo, R., \& Redondo, J. M. (2007). Variables y factores asociados al aprendizaje escolar. Una discusión desde la investigación actual. Estudios Pedagógicos, 33(2), 155-175.

Creswell, J. W. (1998). Qualitative inquiry and research design. Choosing among five traditions. California, United States of America: SAGE Publications, Inc.

Duhart, D. (2004). Juventud Rural en Chile: ¿Problema o Solución? Última década, 12, 121-146.

Echarri, F., \& Puig I Baguer, J. (2008). Environmental education and meaningful learning. Seguridad y Medio Ambiente, 28(112), 1-23.

Echeverría, R. G. (2000). Opciones para reducir la pobreza rural en América Latina y El caribe. Revista CEPAL, 70, 147-160.

Edwards, C. P., Knoche, L., Aukrust, V., Asiye, K., \& Kim, M. (2006). Parental Ethnotheories of Child Development: Looking Beyond Independence and Individualism in American Belief Systems. In U. Kim, K.-S. Yang \& K.-K. Hwang (Eds.), Indigenous and Cultural Psychology. Understanding People in Context (pp. 141-162). USA: Springer Science.

Engeström, Y., \& Sannino, A. (2012). Whatever happened to process theories of learning? Learning, Culture and Social Interaction, 1(1), 45-56.

Erazo, M. S., Villalta, M., \& Morales, M. (2010). Educación básica rural chilena. Realidad y desafíos para la gestión asociativa municipal. Investigaciones en Educación, 10(2), 13-33.

Fernández Droguett, F. (2005). El currículum en la educación intercultural bilingüe: Algunas reflexiones acerca de la diversidad cultural en la educación. Cuadernos Interculturales, 3(4), $7-25$.

Flores, R. (2009). Observando Observadores: Una Introducción a las Técnicas Cualitativas de Investigación Social. Santiago, Chile: Ediciones Universidad Católica de Chile.

Gaínza, A. (2006). La entrevista en profundidad individual. En M. Canales (Ed.), Metodologías de investigación social. Introducción a los oficios (pp. 219-263). Santiago, Chile: Lom Ediciones.

Gibson, J. J. (1986). The ecological approach to visual perception. New York, USA: Taylor \& Francis Group.

Gilmartín, M. Á. (2009). Ambientes escolares. En J. I. Aragonés y M. Amérigo (Eds.), Psicología ambiental (2da ed., pp. 221-237). Madrid, España: ediciones Pirámide.

Hargreaves, L. (2009). Respect and responsibility: Review of research on small rural schools in England. International Journal of Educational Research, 48, 117-128.

Hargreaves, L., Kvalsund, R., \& Galton, M. (2009). Review of research on rural schools and their communities in British and Nordic countries: Analytical perspectives and cultural meaning. International Journal of Educational Research, 48, 80-88.

Holahan, C. J. (2008). Psicología ambiental. Un enfoque general. México D.F.: Editorial Limusa S.A.

Huitt, W. (2006). Overview of classroom processes. Educational Psychology Interactive. Retrieved from http://www.edpsycinteractive.org/topics/process/class.html

Kvalsund, R., \& Hargreaves, L. (2009). Review of research in rural schools and their communities: Analytical perspectives and a new agenda. International Journal of Educational Research, 
48(2), 140-149.

Lakin, M., \& Gasperini, L. (2004). La educación básica en las áreas rurales: situación, problemática y perspectivas. En FAO, UNESCO y IIPE (Eds.), Educación para el desarrollo rural: hacia nuevas respuestas de política (pp. 81-192). Roma, Italia: Ediciones UNESCO, Instituto Internacional de Planeamiento de la Educación.

Maturana, H., \& Varela, F. (2002). El árbol del conocimiento. Las bases biológicas del entendimiento humano (16ta ed.). Santiago, Chile: Editorial Universitaria.

McEwan, P. J. (1998). The effectiveness of multigrade schools in Colombia. International Journal of Educational Development, 18(6), 435-452.

Mizala, A., González, P., Romaguera, P., \& Guzmán, A. (2000). Los Maestros en Chile: Carreras e Incentivos. Washington, D.C.: Banco Interamericano de Desarrollo.

Moreno, C. R. (2007). Las escuelas rurales en Chile: la municipalización y sus fortalezas y debilidades. Revista Digital eRural, Educación, cultura y desarrollo rural, 4(8). Recuperado de www.revistaerural.cl/ojs/index.php/RER/article/download/27/22

Novo, M. (1995). La educación ambiental. Bases éticas, conceptuales y metodológicas. Madrid, España: Editorial Universitas S.A.

Páramo, P. (2007). El significado de los lugares públicos para la gente de Bogotá. Bogotá, Colombia: Universidad Pedagógica Nacional.

Páramo, P. (2010). Aprendizaje situado: creación y modificación de prácticas sociales en el espacio público urbano. Psicologia \& Sociedade, 22(1), 130-138.

Patton, M. Q. (2002). Qualitative Research \& Evaluation Methods (3ra ed.). United States of America: SAGE Publications, Inc.

Rodríguez, G., Gil, J., \& García, E. (1999). Metodología de la investigación cualitativa (2da ed.). Málaga, España: Aljibe, S.L.

Rudduck, J., \& Flutter, J. (2004). How To Improve Your School. Giving Pupils A Voice. New York, USA: CONTINUUM.

Ruíz de Miguel, C. (2001). Factores familiares vinculados al bajo rendimiento. Revista Complutense de Educación, 12(1), 81-113.

Schafft, K. A., \& Jackson, A. Y. (2010). Introduction: Rural Education and Community and TwentyFirst Century. En K. A. Schafft \& A. Y. Jackson (Eds.), Rural Education for the Twenty-First Century. Identity, Place and Community in a Globalizing World. Pennsylvania, United States of America: The Pennsylvania State University Press.

Schiefelbein, E., \& Simmons, J. (1981). Los determinantes del rendimiento escolar: reseña de la investigación para los países en desarrollo. Bogotá, Colombia: IDRC.

Stables, A. (2007). Is Nature Immaterial? The Possibilities for Environmental Education Without an Environment. Canadian Journal of Environmental Education, 12, 55-67.

Stiegler, U. (2008). ¿De la asimilación a la aceptación del otro? política educativa para pueblos indígenas en América Latina y la política de "Educación Intercultural Bilingüe" en Chile. Teoría de la Educación. Educación y Cultura en la Sociedad de la Información, 9(2), 52-76.

Strauss, A., \& Corbin, J. (1998). Basics of Qualitative Research. Techniques and Procedures for Developing Grounded Theory (2da ed.). California, United States of America: SAGE Publications, Inc.

Travers, R. (1976). Fundamentos del aprendizaje. Madrid, España: Santillana S.A.

UNESCO. (2009). Invertir en la diversidad cultural y el dialogo intercultural. Informe mundial de la UNESCO (Resumen). París, Francia: Organización de las Naciones Unidas para la Educación, la Ciencia y la Cultura.

Vera Bachmann, D., Osses, S., \& Schiefelbein, E. (2012). Las Creencias de los profesores rurales: una tarea pendiente para la investigación educativa. Estudios pedagógicos, 38, 297-310.

Villarroel, G., \& Leiva, P. (2005). Emoción y aprendizaje: un estudio en estudiantes de Educación Básica Rural. Revista Digital eRural, Educación, cultura y desarrollo rural, 8(4). 
Estudios Pedagógicos XLII, Nº 3: 111-128, 2016

FACTORES E INTERACCIONES DEL PROCESO DE ENSEÑANZA-APRENDIZAJE EN CONTEXTOS RURALES DE LA

ARAUCANÍA, CHILE

Williamson, G. (2004). Estudio sobre la educación para la población rural en Chile. En FAOUNESCO (Ed.), Educación para la población rural en Brasil, Chile, Colombia, Honduras, México, Paraguay y Perú (pp. 92-163). Roma, Italia.

Zárate, J. (2002). El arte de la relación maestro alumno en el proceso enseñanza aprendizaje. México D.F.: Instituto Politécnico Nacional. 